\title{
DIFFERENTIAL DIMENSION POLYNOMIALS OF FINITELY GENERATED EXTENSIONS
}

\author{
WILLIAM SIT ${ }^{1}$
}

\begin{abstract}
Let $\mathcal{G}=\mathscr{F}\left\langle\eta_{1}, \ldots, \eta_{n}\right\rangle$ be a finitely generated extension of a differential field $\mathscr{F}$ with $m$ derivative operators. Let $d$ be the differential dimension of $\mathcal{G}$ over $\mathcal{F}$. We show that the numerical polynomial

$$
\omega_{\eta / \Phi}(X)-d(\underset{m}{X+m})
$$

can be viewed as the differential dimension polynomial of certain extensions. We then give necessary and sufficient conditions for this numerical polynomial to be zero. An invariant (minimal) differential dimension polynomial for the extension $\mathcal{G}$ over $\mathscr{F}$ is defined and extensions for which this invariant polynomial is $d(\underset{m}{X+M})$ are characterised.
\end{abstract}

Let $\mathcal{G}$ be a finitely generated differential field extension of a differential field $\mathscr{F}$ of characteristic zero with a set $\Delta$ of $m$ commuting derivative operators $\delta_{1}, \ldots, \delta_{m}$. Let $\eta=\left(\eta_{1}, \ldots, \eta_{n}\right)$ be a fixed set of generators of $\mathcal{G}$ over $\mathscr{F}$, that is, $\mathcal{G}=\mathscr{F}\left\langle\eta_{1}, \ldots, \eta_{n}\right\rangle$. The differential dimension polynomial $\omega_{\eta / f}(X)$ was first defined by Kolchin [1] and has the property that for sufficiently large natural numbers $s, \omega_{\eta / f}(s)$ is the transcendence degree over $\mathscr{F}$ of the field extension generated by all derivatives of $\eta_{1}, \ldots, \eta_{n}$ of order up to and including $s$. The polynomial $\omega_{\eta / \text { of }}(X)$ carries with it certain differential birational invariants of the extension $\mathcal{G}$ over $\mathscr{F}$. Writing

$$
\omega_{\eta / \mathscr{F}}(X)=\sum_{i=0}^{m} a_{i}\left(\begin{array}{c}
X+i \\
i
\end{array}\right) \text {, }
$$

these well-known invariants are $a_{m}$ (the differential dimension $d$ of $\mathcal{G}$ over $\mathscr{F}$ ), $\tau$ (the degree of $\omega_{\eta / \mathcal{F}}(X)$, called the differential type of $\mathcal{G}$ over $\mathscr{F}$ ) and $a_{\tau}$ (the typical differential dimension of $\mathcal{G}$ over $\mathscr{F}$ ). Several works in differential algebra are concerned with various properties of the differential dimension polynomial or bounds on the coefficients. See for example Kolchin [1], Johnson [2], [3], Lando [4], Tomasovic [5] and Sit [6], [7].

In this paper, we shall give an interpretation of the numerical polynomial

$$
\omega_{\eta / \mathscr{F}}(X)-d\left(\begin{array}{c}
X+m \\
m
\end{array}\right)
$$

Received by the editors February 9, 1977.

AMS (MOS) subject classifications (1970). Primary 12H05.

Key words and phrases. Differential dimension polynomials, characteristic sets, differential prime ideals, differential polynomials, ranking, initial subsets.

1 This research was supported in part by the Research Foundation of the City University of New York, Grant RF - 11114. 
which we henceforth denote by $\beta_{\eta / g}(X)$. We also show that a necessary and sufficient condition for $\beta_{\eta / f}(X)$ to be zero is that the defining differential ideal of $\eta$ be generated by differential polynomials of order zero. Finally we shall restate the well-ordering theorem in [7] in the context of differential algebra, introduce an invariant differential dimension polynomial for the extension $\mathcal{G}$ over $\mathcal{F}$ and discuss briefly some of its properties.

Proposition 1. Suppose $\eta_{1}, \ldots, \eta_{d}$ form a differential transcendence basis of $\mathcal{G}$ over $\mathcal{F}$. Then

$$
\omega_{\eta_{d+1}, \ldots, \eta_{n} / \mathscr{F}\left\langle\eta_{1}, \ldots, \eta_{d}\right\rangle}(X) \leqslant \beta_{\eta / \mathscr{F}}(X) .
$$

Proof. If $d=0, \omega_{\eta / \mathscr{G}}(X)=\beta_{\eta / \Im}(X)$ and there is nothing to prove. Assume $d \geqslant 1$. As usual, let $\Theta$ (resp. $\Theta(s), s \in \mathbf{N}$ ) be the set of all $\theta=\delta_{1}^{e_{1}} \cdots \delta_{m}^{e_{m}}$ with $\left(e_{1}, \ldots, e_{m}\right) \in \mathbf{N}^{m}$ (resp. all such $\theta$ with $e_{1}+\cdots+e_{m} \leqslant s$ ). Denote the families of elements

$$
\left(\theta \eta_{j}\right)_{\theta \in \Theta(s), 1<j<d^{\prime}}\left(\theta \eta_{j}\right)_{\theta \in \Theta(s), 1<j<n} \text { and }\left(\theta \eta_{j}\right)_{\theta \in \Theta(s), d+1<j<n}
$$

respectively by $\Gamma_{1}^{s}, \Gamma_{2}^{s}$ and $\Gamma_{3}^{s}$. For sufficiently big $s \in \mathbf{N}$, we have

$$
\begin{aligned}
\omega_{\eta_{d+1}, \ldots, \eta_{n} / \mathscr{F}\left\langle\eta_{1}, \ldots, \eta_{d}\right\rangle}(s) & =\operatorname{tr} \operatorname{deg}_{\mathscr{F}\left\langle\eta_{1}, \ldots, \eta_{d}\right\rangle} \mathscr{F}\left\langle\eta_{1}, \ldots, \eta_{d}\right\rangle\left(\Gamma_{3}^{s}\right) \\
& \leqslant \operatorname{tr} \operatorname{deg}_{\mathscr{F}\left(\Gamma_{1}^{s}\right)} \mathscr{F}\left(\Gamma_{1}^{s}, \Gamma_{3}^{s}\right) \\
& =\operatorname{tr} \operatorname{deg}_{\mathscr{F}\left(\Gamma_{1}^{s}\right)} \mathscr{F}\left(\Gamma_{2}^{s}\right) \\
& =\operatorname{tr} \operatorname{deg}_{\mathscr{F}} \mathscr{F}\left(\Gamma_{2}^{s}\right)-\operatorname{tr} \operatorname{deg}_{\mathscr{F}} \mathscr{F}\left(\Gamma_{1}^{s}\right) \\
& =\omega_{\eta / \mathscr{F}}(s)-d\left(\begin{array}{c}
s+m \\
m
\end{array}\right) \\
& =\beta_{\eta / \mathscr{F}}(s) .
\end{aligned}
$$

COROLlary. Let $\tau=\operatorname{deg} \beta_{\eta / \mathscr{F}}$ Suppose $\eta_{1}, \ldots, \eta_{d}$ is a differential transcendence basis of $\mathcal{G}$ over $\mathcal{F}$. Then the differential type of $\mathcal{G}$ over $\mathcal{F}\left\langle\eta_{1}, \ldots, \eta_{d}\right\rangle$ is $\leqslant \tau$ and if it is $\tau$, then the typical differential dimension of $\mathcal{G}$ over $\mathscr{F}\left\langle\eta_{1}, \ldots, \eta_{d}\right\rangle$ is $\leqslant a_{\tau}$.

THEOREM 1. There exists a differential transcendence basis $\eta_{1}, \ldots, \eta_{d}$ (after permuting the $\eta$ 's if necessary) of $\mathcal{G}$ over $\mathscr{F}$ such that

$$
\omega_{\eta_{d+1}, \ldots, \eta_{n} / \mathscr{F}\left\langle\eta_{1}, \ldots, \eta_{d}\right\rangle}(X)=\beta_{\eta / \mathscr{F}}(X) .
$$

Proof. We fix an orderly ranking in $\mathscr{F}\left\{y_{1}, \ldots, y_{n}\right\}$ and let $\mathfrak{p}$ be the defining differential ideal of $\eta_{1}, \ldots, \eta_{n}$ in $\mathscr{F}\left\{y_{1}, \ldots, y_{n}\right\}$. Let $\mathrm{A}$ be a characteristic set of $\mathfrak{p}$. For each $j(1 \leqslant j \leqslant n)$ let $E_{j}$ denote the set of lattice points $\left(e_{1}, \ldots, e_{m}\right) \in \mathbf{N}^{m}$ such that $\delta_{1}^{e_{1}} \cdots \delta_{m}^{e_{m} y_{j}}$ is a leader of an element of A. It was shown in [1, Chapter II, Theorem 6] that

$$
\omega_{p}(X)=\sum_{j=1}^{n} \omega_{E_{j}}(X)
$$

where $\operatorname{deg} \omega_{E_{j}}(X)<m$ if $E_{j} \neq \varnothing$ and $\omega_{E_{j}}(X)=\left(\begin{array}{c}X+m \\ m\end{array}\right)$ if $E_{j}=\varnothing$. Since $\omega_{p}(X)$ $=\omega_{\eta}(X)=d\left(\begin{array}{c}X+m \\ m\end{array}\right)+$ terms of degree less than $m$, we conclude that $E_{j}=\varnothing$ 
for precisely $d$ indices, say for $j=1, \ldots, d$, and that the leader of any element of $\mathrm{A}$ is a derivative of some $y_{k}(d<k<n)$. It follows that $\eta_{1}, \ldots, \eta_{d}$ form a differential transcendence basis of $\mathcal{G}$ over $\mathcal{F}$. By Proposition 1, it suffices to show that for sufficiently large $s$,

$$
\begin{aligned}
& \beta_{\eta / \mathscr{F}}(s)=\operatorname{tr} \operatorname{deg}_{\mathscr{F}\left(\Gamma_{1}^{\mathfrak{s}}\right)} \mathscr{F}\left(\Gamma_{2}^{s}\right) \\
&\left.\leqslant \operatorname{tr} \operatorname{deg}_{\mathscr{F}\left\langle\eta_{1}\right.}, \ldots, \eta_{d}\right\rangle \\
& \mathcal{F}\left\langle\eta_{1}, \ldots, \eta_{d}\right\rangle\left(\Gamma_{3}^{s}\right)
\end{aligned}
$$

(for definitions of $\Gamma_{i}^{s}$, see proof of Proposition 1).

Suppose for some large $s$, the above inequality does not hold, that is, that there exists a set $B$ of derivatives of $y_{d+1}, \ldots, y_{n}$ of order $\leqslant s$ such that $(v(\eta))_{v \in B}$ is algebraically independent over $\mathscr{F}\left(\Gamma_{1}^{s}\right)$ but not algebraically independent over $\mathcal{F}\left\langle\eta_{1}, \ldots, \eta_{d}\right\rangle$. Let $q$ be the smallest natural number $k$ such that $(v(\eta))_{v \in B}$ is algebraically dependent over $\mathscr{F}\left(\Gamma_{1}^{k}\right)$. Then $q>s$ and there exists an $f \in \mathcal{F}\left(\Gamma_{1}^{q}\right)\left[(v)_{v \in B}\right], f \neq 0$, which vanishes at $(v(\eta))_{v \in B}$. Clearing the denominators of $f$, we get a nonzero differential polynomial

$$
P \in \mathscr{F}\left[\left(\theta y_{j}\right)_{\theta \in \Theta(q), 1<j<d} B\right]
$$

such that $P(\eta)=0$. Choose such a $P$ with lowest rank possible. Then the leader $u_{P}$ of $P$ must be of the form $\theta_{1} y_{k}$ with ord $\theta_{1}=q$ and $1 \leqslant k \leqslant d$. Moreover, since the separant $S_{P}$ of $P$ has lower rank than $P, S_{P}(\eta) \neq 0$.

Write $P=\Sigma g_{M} M$ where $M$ runs over a finite set $\Gamma$ of monomials in the indeterminates $(v)_{v \in B}$ and for all $M, g_{M} \neq 0$ and $g_{M} \in \mathscr{F}\left[\left(\theta_{j}\right)_{\theta \in \Theta(q), 1<j<d}\right]$. Clearly, $g_{M}$ is reduced with respect to A. By [1, Proposition 2, p. 80], there exist differential polynomials $\left(M^{\prime}\right)_{M \in \Gamma}$ reduced with respect to $\mathrm{A}$ and of rank no higher than the highest of the ranks of $(M)_{M \in \Gamma}$ (and hence certainly of lower rank than $\left.u_{P}\right)$ and there exists $H \notin \mathfrak{p}(H$ being a product of initials and separants of $A \in \mathrm{A}$ ) such that for all $M \in \Gamma$,

$$
H M \equiv M^{\prime} \bmod [\mathrm{A}] \text {. }
$$

It follows that the differential polynomial $P^{\prime}=\Sigma_{M \in \Gamma} g_{M} M^{\prime}$ is reduced with respect to A. Furthermore, $P \in \mathfrak{p}$ and $H P \equiv P^{\prime} \bmod [\mathrm{A}]$ so that $P^{\prime} \in \mathfrak{p}$. Since $\mathfrak{p}$ contains no nonzero element reduced with respect to $\mathrm{A}, P^{\prime}=0$.

Now, $\partial M^{\prime} / \partial u_{P}=0$ and $\partial M / \partial u_{P}=0$. Hence

$$
\begin{aligned}
H S_{P} & =H \cdot \sum_{M \in \Gamma} \frac{\partial g_{M}}{\partial u_{P}} \cdot M \\
& \equiv \sum_{M \in \Gamma} \frac{\partial g_{M}}{\partial u_{P}} \cdot M^{\prime} \quad \bmod [\mathrm{A}] \\
& \equiv \frac{\partial P^{\prime}}{\partial u_{P}} \bmod [\mathrm{A}] \\
& \equiv 0 \quad \bmod [\mathrm{A}] .
\end{aligned}
$$

Thus $H S_{P} \in \mathfrak{p}$ and $S_{P} \in \mathfrak{p}$. This contradicts $S_{P}(\eta) \neq 0$ and proves the theorem.

THEOREM 2. Let $\eta_{1}, \ldots, \eta_{n}$ be elements in an extension of $\mathscr{F}$, let $V$ be a set 
of derivatives of $y_{1}, \ldots, y_{n}$ and for $s \in \mathbf{N}$, let $V(s)$ be the elements of $V$ of order $\leqslant$. Suppose for some $t \in \mathbf{N}$, we have

(1) $\mathscr{F}\left(\left(\theta \eta_{j}\right)_{\theta \in \Theta(t), 1 \leqslant j \leqslant n}\right)$ is algebraic over $\mathscr{F}\left((v(\eta))_{v \in V(t)}\right)$ and

(2) there ts a $\delta \in \Delta$ such that $(v(\eta))_{v \in V(t) \cup \delta V(t)}$ is algebraically independent over $\mathcal{F}$.

Then $\mathscr{F}\left(\eta_{1}, \ldots, \eta_{n}\right)$ is algebraic over $\mathscr{F}\left((v(\eta))_{v \in V(0)}\right)$. In particular, if Card $V(0)=k$, then Card $V(t) \leqslant k\left(\begin{array}{c}t+m \\ m\end{array}\right)$ and $\omega_{\eta / g}(X) \leqslant k\left(\begin{array}{c}X+m \\ m\end{array}\right)$.

Proof. We first show that $\mathscr{F}\left(\eta_{1}, \ldots, \eta_{n}\right)$ is algebraic over $\mathscr{F}\left(v(\eta)_{v \in V(0)}\right)$. Let $h$ be the smallest natural number $s$ such that $\mathscr{F}\left(\left(\theta \eta_{j}\right)_{\theta \in \Theta(s), 1<j \leqslant n}\right)$ is algebraic over $\mathscr{F}\left((v(\eta))_{v \in V(s)}\right)$. Then $h \leqslant t$. If $h=0$, there is nothing to prove. Suppose $h \geqslant 1$, we shall show that this would lead to a contradiction.

By our choice of $h$, for some derivative $w \in \cup_{j=1}^{n} \Theta(h-1) y_{j}-V(h-1)$, $w(\eta)$ is not algebraic over $\mathscr{F}\left((v(\eta))_{v \in V(h-1)}\right)$. There exists an $f=\sum_{i=0}^{e} g_{i} X^{i} \in$ $\mathscr{F}[V(h), X]$ such that

(i) $g_{i} \in \mathscr{F}[V(h)], g_{e} \neq 0$ and $\left(g_{0}, \ldots, g_{e}\right)=1$,

(ii) $g_{e}\left((v(\eta))_{v \in V(h)}\right)^{-1} f\left((v(\eta))_{v \in V(h)}, X\right)$ is the minimal (monic) polynomial of $w(\eta)$ over $\mathscr{F}\left((v(\eta))_{v \in V(h)}\right)$,

(iii) for some derivative $u \in V(h)-V(h-1), \partial f / \partial u \neq 0$.

For any $g \in \mathscr{F}\left\{y_{1}, \ldots, y_{n}\right\}[X]$ denote $\partial g / \partial u$ by $g^{\prime}$. We claim that $f^{\prime}\left((v(\eta))_{v \in V(h)}, w(\eta)\right) \neq 0$.

Suppose the contrary were true. From (iii) and the assumption that $(v(\eta))_{v \in V(h)}$ are algebraically independent over $\mathscr{F}$, it would follow that $f^{\prime}\left((v(\eta))_{v \in V(h)}, X\right)$ is a nonzero polynomial of degree $\leqslant e$ in $\mathscr{F}\left(\left(v(\eta)_{v \in V(h)}[X]\right.\right.$ that vanishes at $w(\eta)$. Hence for some $\sigma \in \mathscr{F}\left((v(\eta))_{v \in V(h)}\right)$, $\sigma \neq 0$, we have

$$
\sigma f\left((v(\eta))_{v \in V(h)}, X\right)=f^{\prime}\left((v(\eta))_{v \in V(h)}, X\right) .
$$

Equating coefficients of $X^{i}$, we have for $i=0, \ldots, e$

$$
\sigma g_{i}\left((v(\eta))_{v \in V(h)}\right)=g_{i}^{\prime}\left((v(\eta))_{v \in V(h)}\right) .
$$

In particular, $g_{i} \neq 0$ implies $g_{i}^{\prime} \neq 0$. Moreover,

$$
g_{i}\left((v(\eta))_{v \in V(h)}\right) g_{e}^{\prime}\left((v(\eta))_{v \in V(h)}\right)=g_{i}^{\prime}\left((v(\eta))_{v \in V(h)}\right) g_{e}\left((v(\eta))_{v \in V(h)}\right)
$$

and hence $g_{i} g_{e}^{\prime}=g_{i}^{\prime} g_{e}$. Since $\operatorname{deg} g_{e}>\operatorname{deg} g_{e}^{\prime}$, there would be an irreducible factor of $g_{e}$ that divides $g_{i}$ for every $i$ with $g_{i} \neq 0$. This would contradict assumption (i) on $f$. Thus our claim is established.

Now we differentiate the relation $f\left((v(\eta))_{v \in V(h)}, w(\eta)\right)=0$ with respect to $\delta$. We have

$$
\begin{gathered}
\sum_{\bar{v} \in V(h)} \frac{\partial f}{\partial \bar{v}}\left((v(\eta))_{v \in V(h)}, w(\eta)\right) \cdot \delta \bar{v}(\eta)+f^{\delta}\left((v(\eta))_{v \in V(h)}, w(\eta)\right) \\
+\frac{\partial f}{\partial X}\left((v(\eta))_{v \in V(h)}, w(\eta)\right) \cdot \delta w(\eta)=0
\end{gathered}
$$

where $f^{\delta}$ is the polynomial obtained by applying $\delta$ to each coefficient of $f$. By 
our claim,

$$
\delta u(\eta) \in \mathcal{F}\left((v(\eta))_{v \in V(h)},(\delta v(\eta))_{v \in V(h), v \neq u}, w(\eta), \delta w(\eta)\right)
$$

Since both $w(\eta)$ and $\delta w(\eta)$ are algebraic over

$$
\mathcal{F}\left((v(\eta))_{v \in V(h)},(\delta v(\eta))_{v \in V(h), v \neq u}\right)
$$

so is $\delta u(\eta)$. This contradicts the algebraic independence hypothesis in the theorem. Thus $h=0$ and we have proved the first part of the theorem.

Permuting the $\eta$ 's if necessary, let $V(0)$ consist of $\eta_{1}, \ldots, \eta_{k}$. For any $s \in \mathbf{N}, \boldsymbol{\theta}^{\prime} \in \Theta(s), \boldsymbol{\theta}^{\prime} \neq 1$ and $1 \leqslant j \leqslant n$, we have

$$
\boldsymbol{\theta}^{\prime} \eta_{j} \in \mathcal{F}\left(\left(\theta \eta_{i}\right)_{\theta \in \Theta(s), 1<i<k}\right)
$$

and hence

$$
\operatorname{tr} \operatorname{deg}_{\mathscr{F}} \mathscr{F}\left(\left(\theta \eta_{j}\right)_{\theta \in \Theta(s), 1 \leqslant j \leqslant n}\right)=\operatorname{tr} \operatorname{deg}_{\mathscr{F}} \mathscr{F}\left(\left(\theta \eta_{i}\right)_{\theta \in \Theta(s), 1<i<k}\right) \leqslant k\left(\begin{array}{c}
s+m \\
m
\end{array}\right) .
$$

The inequalities stated in the theorem now follow trivially.

Corollary 1. Let $\eta_{1}, \ldots, \eta_{n}$ be elements of an extension of $\mathcal{F}$. Then $\omega_{\eta / F}(X)=d\left(\begin{array}{c}X+m \\ m\end{array}\right)$ if and only if

(*) $\quad \operatorname{diff} \operatorname{dim}_{\mathscr{F}} \mathscr{F}\left\langle\eta_{1}, \ldots, \eta_{n}\right\rangle=\operatorname{tr} \operatorname{deg}_{\mathscr{F}} \mathscr{F}\left(\eta_{1}, \ldots, \eta_{n}\right)=d$.

Proof. Suppose (*) holds. Let $\eta_{1}, \ldots, \eta_{d}$ (renumbering if necessary) be a differential transcendence basis of $\mathscr{F}\left\langle\eta_{1}, \ldots, \eta_{n}\right\rangle$ over $F$. Then $\mathscr{F}\left(\eta_{1}, \ldots, \eta_{n}\right)$ is algebraic over $\mathscr{F}\left(\eta_{1}, \ldots, \eta_{d}\right)$ and as shown in the proof of Theorem 2, $\omega_{\eta / F}(X)=d\left(\begin{array}{c}X+m \\ m\end{array}\right)$.

Conversely suppose $\omega_{\eta / g}(X)=d\left(\begin{array}{c}X+m \\ m\end{array}\right)$. Then

$$
\operatorname{diff} \operatorname{dim}_{\mathscr{F}} \mathscr{F}\left\langle\eta_{1}, \ldots, \eta_{n}\right\rangle=d \text {. }
$$

Let $\eta_{1}, \ldots, \eta_{d}$ be a differential transcendence basis. Applying Theorem 2 to the set $V$ consisting of derivatives $\left(\theta y_{j}\right)_{\theta \in \Theta, 1 \leqslant j \leqslant d}$ we see that $\mathscr{F}\left(\eta_{1}, \ldots, \eta_{n}\right)$ is algebraic over $\mathscr{F}\left(\eta_{1}, \ldots, \eta_{d}\right)$. Hence $\operatorname{tr} \operatorname{deg}_{\mathscr{F}} \mathscr{F}\left(\eta_{1}, \ldots, \eta_{n}\right)=d$.

COROllaRy 2. Let $\mathfrak{p}$ be a prime differential ideal in $\mathscr{F}\left\{y_{1}, \ldots, y_{n}\right\}$ and suppose $\omega_{\mathfrak{p}}(X)=d\left(\begin{array}{c}X+m \\ m\end{array}\right)$. Then $\mathfrak{p}=\left\{\mathfrak{p}_{0}\right\}$ where $\mathfrak{p}_{0}=\mathfrak{p} \cap \mathscr{F}\left[y_{1}, \ldots, y_{n}\right]$ is a prime ideal of dimension $d$.

REMARK. This is a converse of a result of Kolchin [1, Chapter IV, §17, Proposition 10].

Proof. Clearly $\mathfrak{p}_{0}$ is a prime ideal. Let $\eta=\left(\eta_{1}, \ldots, \eta_{n}\right)$ be a generic zero of $\mathfrak{p}$. Then $\eta$ is a generic zero of $\mathfrak{p}_{0}$, and hence by Corollary $1, \operatorname{dim} \mathfrak{p}_{0}=d$. By Kolchin's result mentioned in the remark, $\left\{\mathfrak{p}_{0}\right\}$ is a prime differential ideal with

$$
\omega_{\left\{\mathfrak{p}_{0}\right\} / \mathfrak{F}}(X)=d\left(\begin{array}{c}
X+m \\
m
\end{array}\right) .
$$

Since $\left\{\mathfrak{p}_{0}\right\} \subset \mathfrak{p}$ and $\omega_{\mathfrak{p}}(X)=d\left(\begin{array}{c}X+m \\ m\end{array}\right)$, we have $\left\{\mathfrak{p}_{0}\right\}=\mathfrak{p}$.

We now restate the well-ordering theorem from [7] and use it to introduce an invariant differential dimension polynomial for the extension $\mathcal{G}$ over $\mathscr{F}$. First we recall some notations. 
A subset $V$ of $\mathbf{N}^{m}$ (when $\mathbf{N}^{m}$ is considered as an ordered set relative to the product order induced by the usual order on $\mathbf{N}$ ) is initial if for every $\mathbf{v}$, $\mathbf{v}^{\prime} \in \mathbf{N}^{m}$ such that $\mathbf{v}^{\prime} \leqslant \mathbf{v}, \mathbf{v} \in V$ implies $\mathbf{v}^{\prime} \in V$. For such $V$, it was shown that there exists a numerical polynomial $\omega_{V}(X)$ such that for every sufficiently large $s \in \mathbf{N}, \omega_{V}(s)$ is the number of points $\left(v_{1}, \ldots, v_{m}\right) \in V$ with $v_{1}+\cdots+v_{m} \leqslant s$. If $\mathcal{V}$ is a finite sequence $V_{1}, \ldots, V_{n}$ of initial subsets $V_{i} \subset \mathbf{N}^{m}$, the numerical polynomial of $\mathfrak{V}$ was defined to be

$$
\omega_{\mathscr{V}}=\omega_{V_{1}}+\cdots+\omega_{V_{n}} \text {. }
$$

Now given a differential prime ideal $\mathfrak{p}$ in $\mathscr{F}\left\{y_{1}, \ldots, y_{n}\right\}$, an orderly ranking of the derivatives and a characteristic set $A$ of $\mathfrak{p}$ relative to the given ranking, we may define $V_{j}(1 \leqslant j \leqslant n)$ as the set of lattice points $\left(v_{1}, \ldots, v_{m}\right) \in \mathbf{N}^{m}$ such that $\delta_{1}^{v_{1}} \cdots \delta_{m}^{v_{m}} y_{j}$ is not a derivative of the leader of any element of $\mathrm{A}$. Clearly $V_{j}$ is initial and if $E_{j}$ is the set of lattice points $\left(e_{1}, \ldots, e_{m}\right) \in \mathbf{N}^{m}$ for which $\delta_{1}^{e_{1}} \cdots \delta_{m}^{e_{m}} y_{j}$ is a derivative of the leader of an element of $\mathrm{A}$, then $\omega_{E_{j}}=\omega_{V_{j}}$ by definition [1, p. 51]. Thus $\omega_{\mathfrak{p}}=\omega_{\mathfrak{V}}$ with $\mathscr{V}=\left\{V_{j}\right\}_{j=1}^{m}$. The theorem below then is a direct consequence of Proposition 5 in [7].

THEOREM 3. Let $\Omega$ be the set of differential dimension polynomials $\omega_{\mathfrak{p}}$ for some $\mathfrak{p}$, where $\mathfrak{p}$ is a differential prime ideal in a differential polynomial ring in a finite number of differential indeterminates over some differential field with a finite set of commuting derivative operators. Then $\Omega$ is well-ordered with respect to the order: $\omega_{\mathfrak{p}} \leqslant \omega_{\mathfrak{p}^{\prime}}$ if and only if $\omega_{\mathfrak{p}}(s) \leqslant \omega_{\mathfrak{p}^{\prime}}(s)$ for all sufficiently large $s \in \mathbf{N}$.

DEFINITION. For any finitely generated differential extension $\mathcal{G}$ of $\mathcal{F}$, we define $\omega_{\mathcal{G} / \mathcal{F}}(X)=\min \omega_{\eta / \mathcal{F}}(X)$ where the minimum is taken over all finite families $\eta$ of generators of $\mathcal{G}$ over $\mathscr{F}$.

EXAMPLE. If $\mathcal{G}$ is a pure differential transcendental extension of differential dimension $d$ over $\mathcal{F}$, then $\omega_{\mathcal{G}} / \mathcal{F}(X)=d\left(\begin{array}{c}X+m \\ m\end{array}\right)$.

THEOREM 4. Suppose $\omega_{\mathcal{G} / \mathcal{G}}(X)=d\left(\begin{array}{c}X+m \\ m\end{array}\right)$. Then for any family $\eta=$ $\left(\eta_{1}, \ldots, \eta_{n}\right)$ that generates $\mathcal{G}$ over $\mathscr{F}, \omega_{\eta / \mathcal{F}}(X)=\omega_{\mathcal{G} / \mathcal{F}}(X)$ if and only if $\operatorname{tr} \operatorname{deg}_{\mathscr{F}} \mathscr{F}\left(\eta_{1}, \ldots, \eta_{n}\right)=d$. For such $\eta$, the defining differential ideal of $\eta$ over $\mathcal{F}$ is generated by differential polynomials of order zero. In particular, $\mathcal{G}$ is an algebraic extension of a pure differential transcendental extension of differential dimension $d$ over $\mathcal{F}$.

Proof. Theorem 4 follows from the corollaries of Theorem 2.

Finally we give another equivalent definition of $\omega_{\mathcal{G} / \mathcal{F}}(X)$ using Theorem 1 . Let $\eta$ generate $\mathcal{G}$ over $\mathcal{F}$ and let $\eta^{0}$ be a subfamily of $\eta$ that forms a differential transcendence basis of $\mathcal{G}$ over $\mathscr{F}$. Let $\eta^{1}$ be the complementary subfamily. Proposition 1 and Theorem 1 then show that

$$
\beta_{\eta / \mathscr{F}}(X)=\max _{\eta^{0}} \omega_{\eta^{1} / \mathscr{F}\left\langle\eta^{0}\right\rangle}(X)
$$

and if we define $\beta_{\mathcal{G} / \mathscr{F}}(X)=\min _{\eta} \beta_{\eta / \mathscr{F}}(X)$ then

$$
\omega_{\mathcal{G} / \mathscr{F}}(X)=d\left(\begin{array}{c}
X+m \\
m
\end{array}\right)+\beta_{\mathcal{G} / \mathscr{F}}(X) .
$$




\section{REFERENCES}

1. E. R. Kolchin, Differential algebra and algebraic groups, Academic Press, New York, 1973.

2. Joseph Johnson, Differential dimension polynomials and a fundamental theorem on differential modules, Amer. J. Math. 91 (1969), 239-248.

3.

4. B. A. Lando, Jacobi's bound for the order of systems of first order differential equations, Trans. Amer. Math. Soc. 152 (1970), 119-135.

5. T. S. Tomasovic, A generalised Jacobi conjecture for arbitrary systems of algebraic differential equations, $\mathrm{Ph}$. D. dissertation, Columbia University, 1976.

6. W. Y. Sit, Typical differential dimension of the intersection of linear differential algebraic groups, J. Algebra 32 (1974), 476-487.

7. $37-45$. , Well-ordering of certain numerical polynomials, Trans. Amer. Math. Soc. 212 (1975),

Department of Mathematics, City College (CUNY), New York, New York 10031 